Vol. 49 (1994) [333-339]

\title{
INTEGRABILITY AND L-CONVERGENCE OF MULTIPLE TRIGONOMETRIC SERIES
}

\section{Chang-Pao Chen}

It is proved that under the following condition, the sum $f$ of the double trigonometric series with coefficients $c_{j k}$ is integrable and the rectangular partial sums $s_{m n}(f, x, y)$ converge to $f$ in $L^{1}$-norm:

$$
\sum_{j=-\infty}^{\infty} \sum_{k=-\infty}^{\infty}(\ln |j|)^{\top}(\ln |k|)^{\top}\left|\Delta_{11} c_{j k}\right|<\infty .
$$

This generalises the corresponding results of Móricz [3]. We also prove that the aforementioned condition is sharp. A more general version of this result is established for double series of orthonormal functions, which generalises Móricz-SchippWade [4]. An extension to higher-dimensions is given.

\section{INTRODUCTION}

Let $T^{2}=\left\{(x, y) \in R^{2}:-\pi \leqslant x, y<\pi\right\}$. Consider the double trigonometric series

$$
\sum_{j=-\infty}^{\infty} \sum_{k=-\infty}^{\infty} c_{j k} e^{i(j x+k y)}
$$

We assume that the coefficients $c_{j k}$ satisfy the following two conditions:

$$
\begin{gathered}
c_{j k} \rightarrow 0 \text { as } \max (|j|,|k|) \rightarrow \infty \\
\sum_{j=-\infty}^{\infty} \sum_{k=-\infty}^{\infty}(\ln |j|)^{\top}(\ln |k|)^{\top}\left|\Delta_{11} c_{j k}\right|<\infty
\end{gathered}
$$

where $\xi^{\top}=\max (1, \xi)$ and $\Delta_{11} c_{j k}=c_{j k}-c_{j, k+1}-c_{j+1, k}+c_{j+1, k+1}$. Let $s_{m n}(x, y)$ denote the rectangular partial sums of series (1.1), that is,

$$
s_{m n}(x, y)=\sum_{|j| \leqslant m|k| \leqslant n} \sum_{j k} e^{i(j x+k y)} .
$$

Received 27 May 1993

This research supported by the National Science Council, Taipei, R.O.C. NSC82-0208-M-007-039.

Copyright Clearance Centre, Inc. Serial-fee code: 0004-9729/94 \$A2.00+0.00. 
If the coefficients $c_{j k}$ are the Fourier coefficients of $f \in L^{1}\left(T^{2}\right)$, then we write $s_{m n}(f, x, y)$ instead of $s_{m n}(x, y)$. Recently, Móricz [3] considered the following two kinds of double series:

$$
\begin{gathered}
\sum_{j=0}^{\infty} \sum_{k=0}^{\infty} \lambda_{j} \lambda_{k} a_{j k} \cos j x \cos k y, \\
\sum_{j=1}^{\infty} \sum_{k=1}^{\infty} a_{j k} \sin j x \sin k y
\end{gathered}
$$

where $\lambda_{0}=1 / 2$ and $\lambda_{j}=1$ for $j \geqslant 1$. He proved:

Theorem . (Móricz [3, Theorems 2, 4 and 5].) Assume that (1.1) is either (1.4) or (1.5). If both of conditions (1.2) and (1.3) are satisfied, then the sum $f$ of series (1.1) is integrable, (1.1) is the Fourier series of $f$, and $\left\|s_{m n}(f)-f\right\|_{1} \rightarrow 0$ as $\min (m, n) \rightarrow \infty$.

This result does not deal with the general case (1.1), in particular, the double cosine-sine series

$$
\sum_{j=0}^{\infty} \sum_{k=1}^{\infty} \lambda_{j} a_{j k} \cos j x \sin k y,
$$

where $\lambda_{j}$ are given as above. The purpose of this paper is to extend the aforementioned result of Móricz to any series of type (1.1). Of course, this result will apply to any of (1.4)-(1.6). Our result reads as follows:

THEOREM 1. If both of (1.2) and (1.3) are satisfied, then the sum $f$ of series (1.1) is integrable, (1.1) is the Fourier series of $f$, and $\left\|s_{m n}(f)-f\right\|_{1} \rightarrow 0$ as $\min (m, n) \rightarrow$ $\infty$.

It should be noticed that condition (1.3) cannot be replaced by

$$
\sum_{j=-\infty}^{\infty} \sum_{k=-\infty}^{\infty}\left\{(\ln |j|)^{\top}\right\}^{1-\varepsilon}\left\{(\ln |k|)^{\top}\right\}^{1-\delta}\left|\Delta_{11} c_{j k}\right|<\infty,
$$

where $\varepsilon, \delta \geqslant 0$ and one of them is greater than 0 . The following is a counterexample to the case $\varepsilon>0$ and $\delta>0$ :

$$
\sum_{j=2}^{\infty} \sum_{k=2}^{\infty} \frac{\sin j x \sin k y}{(\ln j)(\ln k)}
$$

Write (1.8) in the form of (1.1) and let $c_{j k}$ be the corresponding coefficients. Then

$$
\left|\Delta_{11} c_{j k}\right| \leqslant M\left(|j|^{\top}|k|^{\top}\right)^{-1}\left\{(\ln |j|)^{\top}(\ln |k|)^{\top}\right\}^{-2}
$$


for all $j$ and for all $k$, where $M$ is an absolute constant. Thus, (1.7) is satisfied for all $\varepsilon>0$ and all $\delta>0$. However $\sum_{j=2}^{\infty}(\sin j x) /(\ln j)$ is not a Fourier series, (see [6, Vol I, p.253]), and so the sum of series (1.8) is not integrable. This says that the conclusion of Theorem 1 fails for this case. On the other hand, we point out that for $\varepsilon, \delta \leqslant 1$ condition (1.7) implies

$$
\sum_{j=-\infty}^{\infty} \sum_{k=-\infty}^{\infty}\left|\Delta_{11} c_{j k}\right|<\infty
$$

Thus, it follows from [2] that the sum $f$ of $(1.1)$ is an $L^{p}\left(T^{2}\right)$-function for all $0<p<1$ and $s_{m n}(x, y)$ converges in the $L^{p}\left(T^{2}\right)$-metric to $f$ as $\min (m, n) \rightarrow \infty$.

As will be pointed out later, the theory developed here can be extended to any double series of the following type:

$$
\sum_{j=0}^{\infty} \sum_{k=0}^{\infty} c_{j k} \phi_{j}(x) \phi_{k}(y),
$$

where $\phi_{j}(t)$ are measurable functions defined on $[0, \alpha)$. The prerequisite is the existence of a sequence $\left\{\Psi_{j}(t): j \geqslant-1\right\}$ of measurable functions such that $\Psi_{-1}(t)=0$,

$$
\begin{array}{ll}
\left|\Psi_{j}(t)\right| \leqslant A(|j|)^{\top} & (j \geqslant 0), \\
\left|\Psi_{j}(t)\right| \leqslant B /|t| & (j \geqslant 0), \\
\Psi_{j}(t)-\Psi_{j-1}(t)=\phi_{j}(t) & (j \geqslant 0),
\end{array}
$$

where $A$ and $B$ are two absolute constants. The precise statement for this is:

THEOREM 2. If (1.2), (1.3) and (1.10)-(1.12) are satisfied, then the sum $f$ of series (1.9) is integrable, and $\left\|s_{m n}(x, y)-f(x, y)\right\|_{1} \rightarrow 0$ as $\min (m, n) \rightarrow \infty$, where

$$
s_{m n}(x, y)=\sum_{j=0}^{m} \sum_{k=0}^{n} c_{j k} \phi_{j}(x) \phi_{k}(y) \quad(m, n \geqslant 0) .
$$

In addition, if $\left\{\phi_{j}(t): j \geqslant 0\right\}$ forms an orthonormal system on $[0, \alpha)$, then

$$
c_{j k}=\int_{0}^{\alpha} \int_{0}^{\alpha} f(x, y) \phi_{j}(x) \phi_{k}(y) d x d y \quad(j, k \geqslant 0) .
$$

Let $\phi_{j}(t)=w_{j}(t)$, where $\left\{w_{j}(t): j \geqslant 0\right\}$ is the Walsh orthonormal system defined on the interval $[0,1)$, in the Paley enumeration. Choose $\Psi_{j}(t)=D_{j}(t)$, the corresponding Walsh-Dirichlet kernel. Then $\Psi_{j}(t)$ possesses the properties (1.10)-(1.12), (see [1] or [5]). Hence, the conclusion of Theorem 2 holds for double Walsh series. This says that Theorem 2 generalises Móricz-Schipp-Wade [4, Theorem 1]. 


\section{Auxiliary lemmas}

Let $\Psi_{j}(t), j=0+, 0-, \pm 1, \pm 2, \ldots$ be the functions defined by: $\Psi_{0+}(t)=$ $\Psi_{0-}(t)=1 / 2$,

$$
\Psi_{j}(t)=\frac{1}{2}+\left(e^{i t}+e^{2 i t}+\ldots+e^{j i t}\right) \quad(j \geqslant 1),
$$

and $\Psi_{-j}(t)=\Psi_{j}(-t)$ for $j \geqslant 1$. Then we have

$$
\left|\Psi_{j}(t)\right| \leqslant \min \left(2|j|^{\top}, \pi /|t|\right) \quad \text { for all } j \text { and for all } t \text {. }
$$

Moreover, for $j \geqslant 0+$, we have

$$
\Psi_{j}(t)=\frac{e^{i(j+1) t}}{2 i e^{i t / 2} \sin t / 2}-\frac{\cos t / 2}{2 i \sin t / 2} \quad(0<|t| \leqslant \pi) .
$$

The next lemma is useful in the proof of the main result.

LemMa 1 . Let $\Psi_{j}(t)$ be defined as above. Then

$$
\int_{-\pi}^{\pi}\left|\Psi_{j}(t)\right| d t \leqslant 6 \pi(\ln |j|)^{\top} \quad \text { for all } j .
$$

Proof of Lemma 1: Let $\rho=|j|^{\top}$. Then by (2.1), we get

$$
\begin{aligned}
\int_{\pi / \rho \leqslant|t| \leqslant \pi}\left|\Psi_{j}(t)\right| d t & \leqslant \pi\left[\int_{\pi / \rho \leqslant|t| \leqslant \pi}(1 /|t|) d t\right] \\
& \leqslant 2 \pi(\ln \rho) \leqslant 2 \pi(\ln |j|)^{\top} .
\end{aligned}
$$

On the other hand, we have

$$
\int_{|t|<\pi / \rho}\left|\Psi_{j}(t)\right| d t \leqslant 4 \pi(|j|)^{\top} / \rho=4 \pi
$$

Combining all that we have done so far, we obtain the desired inequality.

From the definition of $\Psi_{j}(t)$, we find that $\Psi_{0+}(t)+\Psi_{0-}(t)=1$, and

$$
\begin{aligned}
\Psi_{j}(t)-\Psi_{j-1}(t) & =e^{i j t} \quad(j \geqslant 2) ; \quad \Psi_{1}(t)-\Psi_{0+}(t)=e^{i t} \\
\Psi_{-j}(t)-\Psi_{-j+1}(t) & =e^{-i j t} \quad(j \geqslant 2) ; \quad \Psi_{-1}(t)-\Psi_{0-}(t)=e^{-i t} .
\end{aligned}
$$

These relations lead us to the following summation by parts formula, which can be proved by a direct calculation. We leave it to the reader. 
LEMma 2.

$$
\sum_{|j| \leq m} a_{j} e^{i j t}=\sum_{|j|=0 \pm}^{m} \Delta a_{j} \Psi_{j}(t)+\sum_{|j|=m} a_{r(j)} \Psi_{j}(t) .
$$

In this lemma, the sum $\sum_{|j|=0 \pm}^{m}$ means $\sum_{0+\leqslant j \leqslant m}+\sum_{-m \leqslant j \leqslant 0-}$. The function $\tau(j)$ is defined by the formulas: $\tau(0+)=1, \tau(0-)=-1, \tau(j)=j+1$ for $j \geqslant 1$, and $\tau(j)=j-1$ for $j \leqslant-1$.

The first-order differences $\Delta_{1} c_{j k}$ and $\Delta_{2} c_{j k}$ are defined by the formulas:

$$
\Delta_{1} c_{j k}=c_{j k}-c_{r(j), k}, \Delta_{2} c_{j k}=c_{j k}-c_{j, r(k)}
$$

for all $j, k=0 \pm, 1,2, \ldots$, where $c_{0+, k}=c_{0-, k}=c_{0 k}$ and $c_{j, 0+}=c_{j, 0-}=c_{j 0}$. The second-order mixed difference $\Delta_{11}^{*} c_{j k}$ is defined by the formula: $\Delta_{11}^{*} c_{j k}=\Delta_{1} \Delta_{2} c_{j k}=$ $\Delta_{2} \Delta c_{j k}$. The symbols $a_{0+}, a_{0-}$, and $\Delta a_{j}$ are defined in the same way.

Performing a double summation by parts (that is, applying Lemma 2 twice), we get another representation of $s_{m n}(x, y)$ as follows:

LEMMA 3.

$$
\begin{aligned}
s_{m n}(x, y)= & \sum_{|j|=0 \pm|k|=0 \pm}^{m} \sum_{11}^{n} \Delta_{j k}^{*} c_{j} \Psi_{j}(x) \Psi_{k}(y) \\
& +\sum_{|j|=0 \pm \pm|k|=n}^{m} \Delta_{1} c_{j, \tau(k)} \Psi_{j}(x) \Psi_{k}(y) \\
& +\sum_{|j|=m|k|=0 \pm} \sum_{|j|=m}^{n} \Delta_{2} c_{\tau(j), k} \Psi_{j}(x) \Psi_{k}(y) \\
& +\sum_{|j|=m \mid=n} \sum_{\tau(j), \tau(k)} \Psi_{j}(x) \Psi_{k}(y) .
\end{aligned}
$$

\section{Proof of Theorems 1 and 2}

PROOF OF THEOREM 1: By comparing the definitions of $\Delta_{11}$ and $\Delta_{11}^{*}$, we find that (1.3) is equivalent to

$$
\sum_{|j|=0 \pm|k|=0 \pm}^{\infty} \sum^{\infty}(\ln |j|)^{\top}(\ln |k|)^{\top}\left|\Delta_{11}^{*} c_{j k}\right|<\infty .
$$

For any $j$ and any $k$, we have

and

$$
\left|\Delta_{1} c_{j k}\right| \leqslant \sum_{|v|=|k|}^{\infty}\left|\Delta_{11}^{*} c_{j v}\right|
$$

$$
\left|\Delta_{2} c_{j k}\right| \leqslant \sum_{|u|=|j|}^{\infty}\left|\Delta_{11}^{*} c_{u k}\right| \text {. }
$$


From here, we see that (3.1) implies both of the following two conditions:

$$
\begin{aligned}
& {\left[\sum_{|j|=0 \pm}^{\infty}(\ln |j|)^{\top}(\ln |k|)^{\top}\left|\Delta_{1} c_{j k}\right|\right] \rightarrow 0 \text { as }|k| \rightarrow \infty,} \\
& {\left[\sum_{|k|=0 \pm}^{\infty}(\ln |j|)^{\top}(\ln |k|)^{\top}\left|\Delta_{2} c_{j k}\right|\right] \rightarrow 0 \text { as }|j| \rightarrow \infty .}
\end{aligned}
$$

Moreover,

$$
\left|c_{j k}\right| \leqslant \sum_{|u|=|j|}^{\infty} \sum_{|v|=|k|}^{\infty}\left|\Delta_{11}^{*} c_{u v}\right|
$$

and so (3.1) implies that

$$
c_{j k}(\ln |j|)(\ln |k|)=o(1) \quad \text { as } \quad \min (|j|,|k|) \rightarrow \infty .
$$

We know that $L^{1}\left(T^{2}\right)$ is complete. By Lemma 1, Lemma 3, and (3.1)-(3.4), we find that the sum $f$ of series (1.1) is integrable, (1.1) is the Fourier series of $f$, and $\left\|s_{m n}(f)-f\right\|_{1} \rightarrow 0$ as $\min (m, n) \rightarrow \infty$. That is what we want.

Proof of THEOREM 2: Following the proof of Lemma 1, we find that conditions (1.10) and (1.11) together imply

$$
\int_{0}^{\alpha}\left|\Psi_{j}(t)\right| d t \leqslant(A \alpha+B)(\ln |j|)^{\top} \quad(\text { all } j) .
$$

It is assumed that condition (1.12) is satisfied. Hence, results analogous to Lemmas 2 and 3 will hold. Applying the same proof as Theorem 1, with minor modification, we conclude that the sum $f$ of series (1.9) is integrable and

$$
\left\|s_{m n}(x, y)-f(x, y)\right\|_{1} \rightarrow 0 \text { as } \min (m, n) \rightarrow \infty .
$$

We assume that $\left\{\phi_{j}(t): j \geqslant 0\right\}$ forms an orthonormal system on $[0, \alpha)$. Then

$$
c_{j k}=\int_{0}^{\alpha} \int_{0}^{\alpha} s_{m n}(x, y) \phi_{j}(x) \phi_{k}(y) d x d y \quad(m \geqslant j \geqslant 0, n \geqslant k \geqslant 0),
$$

and so by (1.10) and (1.12), we get

$$
\begin{aligned}
\mid c_{j k} & -\int_{0}^{\alpha} \int_{0}^{\alpha} f(x, y) \phi_{j}(x) \phi_{k}(y) d x d y \mid \\
& \leqslant \int_{0}^{\alpha} \int_{0}^{\alpha}\left|s_{m n}(x, y)-f(x, y)\right| \cdot\left|\phi_{j}(x) \phi_{k}(y)\right| d x d y \\
& \leqslant\left(4 A^{2}|j|^{\top}|k|^{\top}\right)\left\|s_{m n}(x, y)-f(x, y)\right\|_{1} \\
& \rightarrow 0 \text { as } \min (m, n) \rightarrow \infty .
\end{aligned}
$$

From here, we get (1.13), and the proof is complete. 
REMARK. Following the above proofs, we find that Theorems 1 and 2 can easily be extended to the higher-dimensional case. In this case, condition (1.3) is replaced by

or

$$
\begin{aligned}
& \sum_{j_{1}=-\infty}^{\infty} \cdots \sum_{j_{n}}^{\infty}\left(\ln \left|j_{1}\right|\right)^{\top} \cdots\left(\ln \left|j_{n}\right|\right)^{\top}\left|\Delta_{1}, \cdots,{ }_{1} c_{j_{1} \cdots j_{n}}\right|<\infty \\
& \sum_{j_{1}=0}^{\infty} \cdots \sum_{j_{n}=0}^{\infty}\left(\ln j_{1}\right)^{\top} \cdots\left(\ln j_{n}\right)^{\top}\left|\Delta_{1}, \cdots,{ }_{1} c_{j_{1}} \cdots j_{n}\right|<\infty .
\end{aligned}
$$

\section{REFERENCES}

[1] N.J. Fine, 'On the Walsh functions', Trans Amer. Math. Soc. 65 (1949), 372-414.

[2] F. Móricz, 'Convergence and integrability of double trigonometric series with coefficients of bounded variation', Proc. Amer. Math. Soc. 102 (1988), 633-640.

[3] F. Móricz, 'On the integrability and $L^{1}$-convergence of double trigonometric series', Studia Math. 88 (1991), 203-225.

[4] F. Móricz, F. Schipp and W.R. Wade, 'On the integrability of double Walsh series with special coefficients', Michigan Math. J. 37 (1990), 191-201.

[5] F. Schipp, W.R. Wade and P. Simon, Walsh Series. An Introduction to Dyadic Harmonic Analysis (Akadémiai Kiadó, Budapest, 1990).

[6] A. Zygmund, Trigonometric Series (Cambridge Univ. Press, 1959).

Department of Mathematics

National Tsing Hua University

Hsinchu

Taiwan 30043

Republic of China 